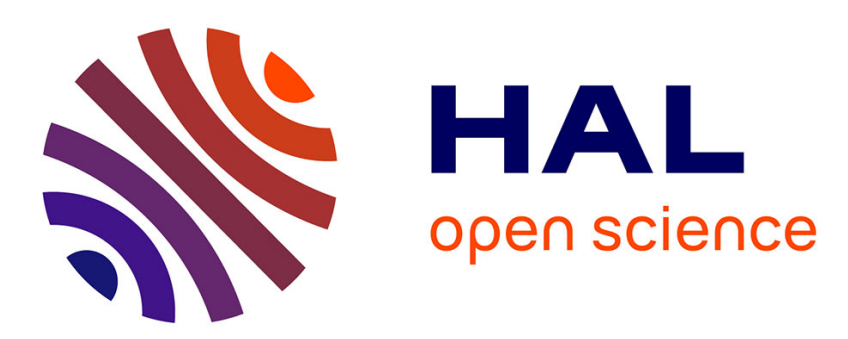

\title{
L' affaire Bordeaux Magnum ou la vente en primeur et la rupture brutale des relations commerciales établies
}

\author{
Ronan Raffray
}

\section{To cite this version:}

Ronan Raffray. L' affaire Bordeaux Magnum ou la vente en primeur et la rupture brutale des relations commerciales établies. Open Wine Law, 2021, 10.20870/owl.2021.4759 . hal-03477391

\section{HAL Id: hal-03477391 \\ https://hal.science/hal-03477391}

Submitted on 13 Dec 2021

HAL is a multi-disciplinary open access archive for the deposit and dissemination of scientific research documents, whether they are published or not. The documents may come from teaching and research institutions in France or abroad, or from public or private research centers.
L'archive ouverte pluridisciplinaire HAL, est destinée au dépôt et à la diffusion de documents scientifiques de niveau recherche, publiés ou non, émanant des établissements d'enseignement et de recherche français ou étrangers, des laboratoires publics ou privés. 


\section{L'affaire Bordeaux Magnum ou la vente en primeur et la rupture brutale des relations commerciales établies}

Par Ronan Raffray, IRDAP, Université de Bordeaux

Commerce de place $v s$ règlementation nationale. La vente de vin constitue un terrain particulièrement favorable à la rencontre du droit du vin et du droit de la concurrence, qu'il s'agisse du prix de vente, des pratiques de vente ou des relations commerciales. Si la vente de vin génère peu de contentieux en proportion des volumes échangés, l'un d'entre eux méritait assurément d'être présenté. Né d'un conflit entre un caviste et des acteurs de la filière bordelaise (grands crus, courtiers, négociants) à propos d'une diminution de ses allocations en primeur, ce contentieux a pour l'essentiel - le refus de vente avait été plaidé, mais avait buté sur le statut de professionnel de l'acheteur ${ }^{1}$ - convoqué les règles protectrices de la concurrence luttant contre la rupture brutale des relations commerciales établies, traitée par le Code de commerce comme une pratique déloyale restrictive de concurrence. A l'époque des faits, le dispositif de l'article L. 442-6, I, $5^{\circ}$ du Code de commerce ${ }^{2}$ prévoyait « qu'engage la responsabilité de son auteur et l'oblige à réparer le préjudice causé le fait, par tout producteur, commerçant, industriel ou personne immatriculée au répertoire des métiers: [...] $5^{\circ}$ De rompre brutalement, même partiellement, une relation commerciale établie, sans préavis écrit tenant compte de la durée de la relation commerciale et respectant la durée minimale de préavis déterminée, en référence aux usages du commerce, par des accords interprofessionnels ». Ce dispositif avait-il vocation à régir les relations commerciales qui se nouent à Bordeaux lors de la campagne des primeurs ? La rencontre d'un système de vente construit sur les usages de place ${ }^{3}$ et d'une règlementation nationale soucieuse de normer la fin des relations commerciales s'annonçait particulièrement instructive ${ }^{4}$.

Logique économique de la vente de(s) vin(s) en primeur. A Bordeaux, la « campagne» des primeurs démarre avec la «semaine » des primeurs, qui réunit des milliers de professionnels de la filière $^{5}$ (experts, journalistes, acheteurs) au début du mois d'avril. Les trois acteurs principaux de cette vente ${ }^{6}$ sont les viticulteurs, qui conduisent la vigne, récoltent le raisin et élèvent le vin, les négociants, qui achètent le vin pour le revendre, et les courtiers, qui rapprochent les deux parties. Lors de cette période décisive de l'année, les négociants vont donc se rapprocher des producteurs de grands crus, par l'intermédiaire des courtiers, en vue d'acheter le vin issu de la dernière vendange. Le vin fait donc l'objet d'une première vente là où il est produit - cette dernière caractéristique faisant du commerce de vin un commerce de place ${ }^{7}$. Une fois qu'il connait son allocation, le

\footnotetext{
${ }^{1}$ CA Bordeaux, $1^{\text {ère }}$ ch., section B, 17 juin 2010, $\mathrm{n}^{\circ}$ 09/01264.

${ }^{2}$ Depuis l'ordonnance n⿳2 2019-359 du 24 avril 2019 portant refonte du titre IV du livre IV du Code de commerce relatif à la transparence, aux pratiques restrictives de concurrence et aux autres pratiques prohibées (JO 25 avr. 2019, texte n 16), le dispositif est inscrit à L. 442-1, II nouveau du Code de commerce, ainsi rédigé : « Engage la responsabilité de son auteur et l'oblige à réparer le préjudice causé le fait, par toute personne exerçant des activités de production, de distribution ou de services de rompre brutalement, même partiellement, une relation commerciale établie, en l'absence d'un préavis écrit qui tienne compte notamment de la durée de la relation commerciale, en référence aux usages du commerce ou aux accords interprofessionnels ».

${ }^{3}$ Pour un exemple de rupture brutale de relations commerciales établies en Bourgogne, v. CA Dijon, 5 juin 2007, $\mathrm{n}^{\circ} 06 / 02191$.

${ }^{4}$ Pour un précédent, v. E. Agostini, «Primeurs en Bordelais », obs. sous CA Bordeaux, 2 ${ }^{\text {ème }}$ ch., 19 févr. 2003, D. 2003, p. 1789. Sur l'arrêt de cassation, v. J.-J. Barbieri et C. Formage, "Sur les "relations commerciales" successives en matière de distribution de vins en primeur », obs. sous Cass. com., 7 juin 2006, n 03-12.659, RD rur. 2006, n 346, comm. 225.

56500 inscrits pour l'année 2016 (chiffres CIVB).

${ }^{6}$ Pour cette description de la vente, $\mathrm{v}$. La vente de vins en primeur, Etude et avis sur la comptabilité et le traitement fiscal des opérations de vente de vins en primeur, 12 juillet 1999, Compagnie régionale des commissaires aux comptes, Bordeaux. ${ }^{7}$ J.-M. Bahans et M. Menjucq, Droit de la vigne et du vin, aspects juridiques du marché viticole, LexisNexis, 3 ème éd., 2020, nos 876 et s.
} 
négociant va pouvoir commercialiser les vins auprès de sa clientèle à qui le vin sera livré après élevage dans les chais du producteur. Un tel schéma permet au producteur de disposer de liquidités et de bénéficier des infrastructures du négoce pour distribuer son vin $^{8}$, tandis qu'il offre aux négociants la possibilité de sécuriser leur approvisionnement et d'effectuer des plus-values rapides. Le consommateur espère quant à lui payer un peu moins cher que s'il attend la sortie du vin, même s'il arrive que le prix de certains vins soit finalement moins élevé que le prix primeurs?. Economiquement, la vente en primeur repose donc sur une logique qui lui est propre et qui puise aux sources de la pratique vitivinicole. Connaissant sa première manifestation, semble-t-il, au $18^{\mathrm{e}}$ siècle lorsque la récolte des crus était mise en bouteille chez les négociants ${ }^{10}$, elle s'est institutionnalisée dans sa configuration moderne à Bordeaux à partir des années 1970. La science économique perçoit le système des primeurs comme une manifestation de la "résistance » des outils commerciaux traditionnels, mettant en avant, d'une part, la logique d'amont qui s'y exprime, le marché devant s'adapter au prix fixé par le producteur ${ }^{11}$, et, d'autre part, son caractère fermé, illustré par la précieuse «allocation $»^{12}$ qui permet au négociant de participer au commerce des "vins de château $»^{13}$. Ce particularisme économique a dicté les contours juridiques et fiscaux ${ }^{14} \mathrm{de}$ la figure de la vente en primeur.

Schéma juridique de la vente en primeur. Pratique ancienne de la place bordelaise ${ }^{15}$, bien installée dans d'autres régions viticoles, la vente en primeur repose sur un schéma juridique connu quant à son déroulement ${ }^{16}$ mais moins abouti sur le plan de certaines qualifications juridiques. Certains contours demeurent incertains, notamment en raison de la rareté du contentieux qui n'a pas entièrement permis à la jurisprudence de réaliser son œuvre de clarification. Une telle vente, fondée sur la confiance et les usages de place, ne se prête pas au duel judiciaire ${ }^{17}$, si bien que les décisions relatives à ce "marché fermés ${ }^{18}$ ne sont pas légion. Si l'on excepte les incertitudes comptables $^{19}$ et le contentieux fiscal ayant donné lieu à la célèbre jurisprudence dite «De

\footnotetext{
8 P.-M. Chauvin, Le marché des réputations, Une sociologie du monde des vins de Bordeaux, Féret, 2010, p. 69.

${ }^{9}$ Ibid.

${ }^{10}$ La vente de vins en primeur, Etude et avis sur la comptabilité et le traitement fiscal des opérations de vente de vins en primeur, étude préc.

11 V. ainsi M.-P. Chauvin, Le marché des réputations, Une sociologie du monde des vins de Bordeaux, op. cit., p. 67.

12 E. Agostini, «Primeurs en Bordelais », art. préc. : «il est vital pour le négoce de bénéficier d'allocations régulières de la part de crus prestigieux qu'il propose à sa clientèle. De la sorte, il fidélise celle-ci ; il soigne sa propre image de marque ; et il assure sa trésorerie ».

${ }^{13} \mathrm{~V}$ ainsi M. Rejalot, Les logiques de château, Filière et modèle viti-vinicole à Bordeaux, 1980-2003, Presses universitaires de Bordeaux, p. 121 et s.

${ }^{14}$ Sur lesquels v. E. Agostini, D. Lencou et B. Pharé, Les primeurs, le courtier et le fisc, note sous CE, 28 juill. 2000 , D. 2001, p. 1419 ; M. Menjucq, «Impromptu sur la TVA de la vente en primeur des grands crus de vins de Bordeaux ", Ecrits de fiscalité des entreprises, Etudes à la mémoire du professeur Maurice. Cozian, Litec, 2009, p. 877 ; T. Droulez et A. Benderdouch, «Ventes de vins en primeur, Le vin est-il bien livré », Revue fiscale du patrimoine, $\mathrm{n}^{\circ}$ 10 , oct. 2020, p. 1.

15 M. Réjalot, Les logiques de château, Filière et modèle viti-vinicole à Bordeaux, op. cit., p. 121 et s. ; O.-A. Geny, D. Geny et M. Roudil, Manuel pratique du commerce des vins, Féret, 2ème éd., 2013, p. 35 et s. ; M.-P. Chauvin, Le marché des réputations. Une sociologie du monde des vins de Bordeaux, op. cit., p. 67 et s.

${ }^{16}$ V. notamment D. Denis, La vigne et le vin, Régime juridique, Sirey, 1985, n 122 ; J.-M. Bahans et M. Menjucq, Droit de la vigne et du vin, aspects juridiques du marché viticole, op. cit., nos 876 et s. ; M. Menjucq, « Le particularisme du droit français de la vente de vins », RLDA 2008, p. 81 et s.; E. Agostini, " Primeurs en Bordelais », D. 2003 , p. 1789 ; S. Crevel, RD rur. 2006, p. 28 ; M. Ruiz, Contrats, conc. consom., $1^{\text {er }}$ décembre 2009.

17 V. toutefois, pour du contentieux : CA Bordeaux, 5 avr. 2001, n RG 99/1020 (évaluation d'un préjudice par application du prix de vente du vin en primeur) ; Cass. crim., 25 sept. 1997, n 96-84.665 (escroquerie et vente de vins en primeur) ; Cass. com., 7 juill. 1998, $\mathrm{n}^{\circ}$ 96-15.296, JCP G 1998, 2176 (vente en primeur et constitution d'un gage).

18 M. Réjalot, Les logiques de château, Filière et modèle viti-vinicole à Bordeaux, op. cit., p. 125.

${ }^{19} \mathrm{~V}$. La vente de vins en primeur, Etude et avis sur la comptabilité et le traitement fiscal des opérations de vente de vins en primeur, étude préc.
} 
Bouärd ${ }^{20}$, peu de jugements et arrêts sont venus éclairer cette opération. Certaines bases sont acquises. Il en est ainsi de l'objet de la vente, que l'on qualifie de chose future ${ }^{21}$ puisque le vin n'a pas fini son élevage, et de chose de genre dès lors qu'il n'a pas été individualisé. Plus difficile est la qualification de l'opération associant deux ventes successives. Par la première vente, le viticulteur, qui a récolté les raisins et s'apprête à élever le vin, s'adresse à des courtiers pour écouler sa production. Le courtier, intermédiaire, proposera le vin à un négociant qui se portera acquéreur d'une certaine quantité de vin. La vente est matérialisée par la signature d'un bordereau de courtage, seul instrumentum mobilisé. L'achat est en général payé par tiers (un tiers à la signature du contrat, un deuxième tiers six mois plus tard, le dernier avant la retiraison des vins). Des règlements par moitié peuvent également être pratiqués au moment de la transaction puis avant la retiraison. Concomitamment est conclue la seconde vente entre le négociant (premier acquéreur), qui interviendra comme vendeur, et un autre négociant (un courtier peut alors être sollicité) ou le client final (sans intervention d'un courtier). Le vin sera livré à la fin des opérations d'élevage, après mise en bouteille et étiquetage. Les vins peuvent être directement livrés aux clients, mais peuvent également être stockés par le négociant, avant toute nouvelle transaction. Financièrement, le négociant appelle souvent des acomptes à ses clients au même rythme que ceux qui lui sont demandés par le château. La vente en primeur s'analyse donc en une opération complexe, au sens d'opération reposant sur la conclusion de plusieurs contrats : le premier contrat entre le producteur et le négociant, conclu par l'intermédiaire du courtier, se double d'un deuxième contrat conclu entre le négociant et son client, professionnel ou consommateur. La qualification juridique de cette succession de contrats peut s'opérer au moyen de plusieurs notions, principalement celles de souscontrat, de cession de contrat, de substitution de contractant et de revente ${ }^{22}$. Les opérations translatives, de cession de contrat ou de substitution de contractant n'offrent pas d'intérêt, car l'existence de deux contrats n'est pas discutée. La qualification de sous-contrat ne permet pas quant à elle de retranscrire fidèlement le processus contractuel ${ }^{23}$ suivi, dans la mesure où le second contrat ne constitue pas un moyen d'exécution du premier ${ }^{24}$. La revente, terme que l'on retrouve dans les modèles professionnels, exprime en revanche avec précision le montage contractuel né de la pratique, lequel associe deux ventes sans toutefois associer le second acquéreur à l'exécution du premier contrat. Outre l'utilisation du terme, la documentation professionnelle ${ }^{25}$ témoigne d'ailleurs de la réalisation de deux ventes successives car chaque acquéreur s'engagea à payer le prix à son vendeur. De même, le premier vendeur n'est engagé qu'à l'égard du premier acquéreur, ce

\footnotetext{
${ }^{20}$ Sur laquelle v. M. Menjucq, «Impromptu sur la TVA de la vente en primeur des grands crus de vins de Bordeaux », art. préc.

${ }^{21}$ J.-M. Bahans et M. Menjucq, Droit de la vigne et du vin, aspects juridiques du marché viticole, op. cit., $\mathrm{n}^{\circ} 884$.

22 R. Bonhomme et M. Bouteille-Brigant, J.-Cl. Contrats Distribution, Fasc. 105 : «Sous-contrat et co-contrat. Adjonction et conjonction de contractants », $\mathrm{n}^{\circ} 8:$ : Le sous-contrat n'est pas une simple succession de contrats de même nature. Le sous-contrat dépend du contrat principal dont il tend à assurer l'exécution. Une simple succession a lieu lorsque les contrats qui se suivent et portent sur le même objet sont indépendants parce que la conclusion du suivant signifie que l'effet principal du précédent s'est réalisé. Il en est ainsi en cas de revente d'un bien par son acquéreur ; il y a là une succession de contrats de vente et non la conclusion d'un sous-contrat ».

${ }^{23}$ V. notamment D. Mainguy, La revente, thèse, Litec, Bibl. dr. entr., t. 35, 1996.

${ }^{24} \mathrm{R}$. Bonhomme et M. Bouteille-Brigant, fasc. préc., $\mathrm{n}^{\circ} 5: \mathrm{Il}$ y a sous-contrat lorsque le second contrat associe les parties à l'exécution du premier. Plus précisément il y a sous-contrat lorsqu'une relation contractuelle s'établit « entre deux personnes (B) et (C) soit en vue de l'exécution par (C) de tout ou partie des obligations auxquelles s'est engagé (B) dans la relation contractuelle qu'il a établie avec (A), soit pour faire bénéficier (C) de l'exécution par (A) de tout ou partie des obligations auxquelles celui-ci s'est engagé au profit de (B). Autrement dit, le souscontrat est un contrat secondaire conclu avec un tiers par l'un des contractants à un contrat principal et destiné à l'exécution de celui-ci (...)». Dans l'hypothèse d'une vente en primeur il y aurait sous-contrat si le second acquéreur participait à l'exécution du premier contrat, c'est-à-dire s'il est rendu créancier de l'obligation de transférer la propriété qui pèse sur le viticulteur ou s'il est débiteur de l'obligation de payer le prix contracte par le premier acquéreur à l'égard du vendeur (le château).

$25 \mathrm{~V}$. La vente de vins en primeur, Etude et avis sur la comptabilité et le traitement fiscal des opérations de vente de vins en primeur, étude préc.
} 
qui exclut une action directe du second contre le premier. Ainsi on trouvera la clause suivante : « en matière de revente, sauf convention contraire, le vendeur n'aura jamais affaire, pour quoi que ce soit, qu'à l'acheteur au nom duquel le présent bordereau est établi ». La revente exprime tout aussi bien la porosité des deux contrats, le producteur conseillant un prix de revente ${ }^{26}$ et pouvant se montrer attentif à la composition de la clientèle de son acheteur. Juridiquement, donc, l'autonomie de chacun des contrats n'est pas discutée. Leur intégration dans une même opération économique n'est pas non plus contestable. C'est ici que la qualification de revente se révèle utile, en ce sens qu'elle exprime l'individualité préservée des deux contrats de l'opération complexe tout en restituant le lien existant entre les deux contrats successifs à raison de leur objet.

Exposé des litiges. Ces dernières années ont vu apparaittre nombre de contentieux qui ont incontestablement enrichi l'étude de cette opération complexe. Un caviste ayant fait l'objet d'une procédure de redressement judiciaire ouverte par jugement du 30 juin 2010, la vente en primeur s'est tout d'abord trouvée confrontée au régime de la continuation des contrats en $\operatorname{cours}^{27}$, ce qui a donné l'occasion à la Cour de cassation de se prononcer sur sa soumission ou non à un tel régime lors de l'ouverture de la procédure collective. Ce caviste bordelais s'est ensuite risqué à attaquer des producteurs consécutivement à la baisse ou à la suppression de ses allocations ${ }^{28}$, ce qui a conduit la jurisprudence à statuer plusieurs fois sur l'application de l'article L. 442-6 du Code de commerce à cette vente si particulière, contentieux qui ne connaissait que peu de précédents en matière viticole $^{29}$. Menées à la fois contre les châteaux, les courtiers et les négociants, les actions ont invité plusieurs cours d'appel à apprécier la spécificité de la vente en primeur et ont contribué à en illustrer la singularité juridique. L'on présentera les arrêts (I) avant d'en tirer quelques enseignements (II).

\section{Les arrêts}

Le caviste avait choisi d'agir sur le même fondement contre plusieurs partenaires économiques : producteurs $(\mathbf{A})$, courtiers $(\mathbf{B})$ et négociants $(\mathbf{C})$. La demande de réparation portait généralement sur une diminution du volume d'affaires à l'occasion des primeurs.

\section{A. Les actions contre les producteurs}

Quatre décisions. Les actions directement menées contre les châteaux ont donné lieu à quatre arrêts, rendus pour deux d'entre eux par la Cour d'appel de Bordeaux en $2009^{30}$ et $2015^{31}$, pour deux autres par la Cour d'appel de Paris en $2015^{32}$. Chaque décision illustre un aspect du régime de

\footnotetext{
26 P.-M. Chauvin, Le marché des réputations, Une sociologie du monde des vins de Bordeaux, op. cit., p. 86.

${ }_{27}$ V. notamment R. Raffray, «Vente en primeur et contrats en cours », obs. sous CA Bordeaux, $2^{\text {ème }}$ ch. civ., 27 févr. 2015, n 12/06029, RPC 2017, p. 27.

${ }^{28}$ L'on portera à la connaissance de nos lecteurs que le propriétaire et dirigeant de la société, champenois d'origine, soulignait dans ses conclusions le fait que la baisse drastique de ces allocations était concomitante de son acquisition de la société.

${ }^{29}$ V. ainsi, à Bordeaux, CA Bordeaux, 2 ème ch., 19 févr. 2003, préc. Pour l'arrêt de cassation, v. Cass. com., 7 juin 2006, n 03-12.659, préc. V. aussi, pour une action identique en bourgogne, CA Dijon, 5 juin 2007, $\mathrm{n}^{\circ}$ 06/0291. V. encore, pour un conflit entre producteur et acheteur, mais hors vente en primeur, CA Versailles, $\mathrm{n}^{\circ} 10 / 08577$ : JurisData $\mathrm{n}^{\circ}$ 2012-016676 : «en donnant au mois de janvier un préavis pour la fin de la période saisonnière annuelle, la société Monoprix a respecté un préavis suffisant au regard des usages et de la durée de la relation commerciale, permettant à la société Escarelle de trouver une solution alternative pour le prochain cycle de production attaché au domaine viticole »). V. enfin, également hors campagne des primeurs, Cass. com., 7 juin 2006, n 03-12.659, préc.; Cass. com., 3 juill. 2019, n 18-10.580 (demande rejetée en l'espèce car la rupture résultait d'un désaccord sur le prix de vente).

${ }^{30}$ CA Bordeaux, $1^{\text {ère }}$ ch. civ., section B, 30 avr. 2009, nº 07/02409.

${ }^{31}$ CA Bordeaux, $2^{\mathrm{ème}}$ ch. civ., 2 juill. 2015, n 10/07300.

${ }^{32}$ CA Paris, Pôle 5, ch. 4, 15 avr. 2015, n 13/02730 : Contrats, conc. consom. 2015, n 7, comm. 174, note N. Mathey ; CA Paris, Pôle 5, ch. 4, 15 avr. 2015, n 13/02675.
} 
responsabilité pour rupture brutale de relation contractuelle établie, qu'il s'agisse de la possibilité de l'action, de l'examen de la brutalité de la rupture ou de l'établissement de la relation contractuelle elle-même.

Premier arrêt : la possibilité de l'action. La lecture du premier arrêt rendu par la Cour d'appel de Bordeaux ${ }^{33}$ nous apprend que le caviste se plaignait que la société civile du château Latour a brutalement cessé de lui allouer les trente caisses qu'elle lui avait allouées annuellement sur une période de 8 années consécutives. La société productrice contestait quant à elle l'existence d'une relation commerciale établie. L'existence de cette relation ne faisait toutefois aucun doute pour la Cour, peu importe le nombre limité de caisses allouées annuellement (30 caisses de douze bouteilles, soit 360 bouteilles par an tout de même), si bien que la société exploitante avait été condamnée à réparer le préjudice sur la base d'une marge annuelle de 1,20 ainsi qu'au paiement d'une indemnité de désorganisation de clientèle. Rappelons tout d'abord qu'il est acquis que l'action peut être menée contre un professionnel civil ${ }^{34}$. La rédaction du texte, qui vise tout producteur, commerçant, industriel ou personne immatriculée au répertoire des métiers, a effectivement permis à la jurisprudence d'en étendre considérablement le domaine au-delà des strictes relations commerciales au sens de la commercialité de l'article L. 110-1 du Code de commerce. Soulignons ensuite qu'il est également acquis que des contrats successifs, qui ont un objet identique, peuvent constituer des « relations commerciales » au sens du Livre IV du Code de commerce ${ }^{35}$, et qu'il n'est donc pas nécessaire pour établir l'existence de telles relations qu'il y ait un accord-cadre ${ }^{36}$. L'annualité de la vente ne constitue pas non plus un problème, la Commission d'examen des pratiques commerciales ayant exclu que le caractère annuel d'une convention puisse écarter a priori toute stabilité de la relation. Il a ainsi été jugé à propos d'une foire se tenant sur quelques jours, une fois par an, qu'il suffisait de prouver une participation à la foire pendant plusieurs années ${ }^{37}$.

Deuxième arrêt : l'examen de la brutalité de la rupture. En remettant en cause la brutalité de la rupture, le deuxième arrêt de la Cour d'appel de Bordeaux ${ }^{38}$ était bien moins favorable au demandeur. Après une longue relation commerciale qui n'était pas contestée par le château, les allocations avaient sensiblement baissé en 2007, si bien que la société Bordeaux Magnum avait engagé une action aux fins de voir, pour l'essentiel, constater la rupture brutale et sans préavis des relations commerciales établies par la société exploitante et obtenir la condamnation de cette dernière à réparer son préjudice. Elle se fondait sur la diminution progressive du volume de ses allocations, qui n'avaient jamais été inférieures à 120 bouteilles, mais ramenées à 48 bouteilles en 2006. L'existence de relations commerciales établies n'était d'ailleurs pas contestée par le défendeur, la discussion portant directement sur la caractérisation de la rupture brutale de la relation commerciale. La société productrice contestait toute rupture fautive des relations commerciales avec la société Bordeaux Magnum, soulignant pour sa défense que le demandeur «a vu ses allocations diminuées progressivement, le château producteur restant libre de décider la quote-part de vins vendus en primeur en fonction des rendements et de la qualité de la récolte, mais également des impératifs de gestion économique de son exploitation ». Pour débouter le demandeur la Cour rappelle tout d'abord que la société Bordeaux Magnum exerce une activité de vente en détail de vins et spiritueux et achète en primeur depuis plusieurs années, bénéficiant ainsi d'«allocations de primeurs » lui permettant d'acheter ce vin aux conditions de sortie des châteaux (...) à des conditions tarifaires intéressantes bien qu'elle ne soit pas négociant ». Reprenant ensuite l'analyse

\footnotetext{
33 CA Bordeaux, 1 ère ch. civ, section B, 30 avr. 2009, nº 07/02409.

${ }^{34}$ C.-A. Maetz, J.-Cl. Concurrence.-Consommation, « Rupture brutale de relations commerciales établies », $\mathrm{n}^{\circ} 15$.

35 Cass. com., 15 sept. 2009, JurisData n 2009-049448.

${ }^{36}$ CA Paris, 7 avr. 2011, RG nº 09-28913.

${ }^{37}$ Cass. com., 15 sept. 2009, $\mathrm{n}^{\circ}$ 08-19.200.

${ }^{38}$ CA Bordeaux, $2^{\mathrm{ème}}$ ch. civ., 2 juill. $2015, \mathrm{n}^{\circ} 10 / 07300$.
} 
proposée au Dalloz par le Professeur Eric Agostini ${ }^{39}$, la Cour constate que les relations commerciales entre les parties ne reposent pas sur un contrat écrit, mais interviennent dans le cadre des ventes de vins en primeur, pratique bordelaise qui implique que «l'allocation de primeurs » reste à la discrétion du viticulteur, ce dernier décidant de la mise en marché en fonction de différents critères d'ordre qualitatif, quantitatif et économique, ce qui autorise la variabilité des quanta alloués d'une année sur l'autre. Il en résulte, selon la Cour, qu'au regard de la spécificité de la vente de vin en primeur, le bénéficiaire de l'allocation ne connaît pas exactement, d'une année sur l'autre, le nombre de bouteilles qui va lui être alloué ni si les demandes qu'il a faites à ce titre vont être honorées. Cette variabilité inhérente à cette vente doit alors être combinée à la rupture brutale des relations commerciales établies, laquelle peut être partielle, mais doit être, selon le droit commun, imprévisible, soudaine et violente et « suppose un effet de surprise pour la victime ». Selon la Cour rien n'obligeait ainsi la société productrice à informer préalablement la société Bordeaux Magnum de la quantité de vin en primeur qui allait lui être accordée, cette décision relevant exclusivement du choix de la ou des propriétés en fonction de la politique de vente adoptée. La rupture brutale de la relation commerciale n'était donc pas caractérisée.

Troisième et quatrième arrêts : l'établissement de la relation contractuelle. Le troisième arrêt ${ }^{40}$ complétait ce dispositif jurisprudentiel en revenant sur la qualification de la relation contractuelle, jugée en l'espèce doublement précaire en considération d'éléments de fait (durée de la relation), et des éléments structurels de cette vente, à savoir le statut professionnel de l'acheteur (caviste et non négociant) et l'aléa climatique. Pour la Cour la durée de la relation (deux campagnes de primeurs) devait en effet être examinée au regard des usages ainsi qu'au regard du produit vendu. Sur le premier point et en considération des usages, la Cour souligne que la vente de vin en primeur est réalisée habituellement par l'intermédiaire de courtiers au profit de négociants, ce que la société Bordeaux Magnum n'est pas. Un tel schéma de vente, inhabituel, donne donc à la relation entre les parties un caractère exceptionnel, et, par-là, précaire. Sur le second point, la Cour considère que la vente de vin en primeur est soumise aux aléas climatiques de sorte que la quantité d'allocations est susceptible de varier d'une année à l'autre en fonction de la quantité produite et de sa qualité. Il ressortait de ces observations que l'allocation accordée était exceptionnelle, sur une période restreinte de deux ans et sur des quantités dégressives, si bien que l'existence d'une relation commerciale régulière et stable entre les parties n'était pas établie. En complément la Cour rejetait également la brutalité de la rupture au constat qu'une lettre du château reprochait au caviste « de ne pas avoir valorisé sa production, de commercialiser les produits dans le but essentiel de se procurer de la trésorerie » et soulignait « que les chiffres d'affaires de la société Bordeaux Magnum s'effondrent», prévenant "que ces faits et le tour que semblent prendre leurs relations étaient susceptibles de compromettre définitivement pour l'avenir la poursuite des rapports commerciaux ». Il est vrai que l'inexécution grave de ses obligations par le cocontractant constitue un fait justificatif de la brutalité de la rupture ${ }^{41}$. Dans un autre litige opposant le caviste à une propriété du même groupe ${ }^{42}$, la Cour de Paris s'appuyait sur des éléments identiques, rappelant que la vente est soumise aux aléas climatiques et que les allocations avaient été accordées sur une période restreinte, sur des qualités minimes et dégressives.

\section{B. Les actions contre les courtiers}

\footnotetext{
${ }^{39}$ E. Agostini, « Primeurs en Bordelais », art. préc.

40 CA, Paris, pôle 5, ch. 4, 15 avr. 2015, n $13 / 02730$.

41 Tel qu'il est aujourd'hui prévu à l'article L. 442-6 du Code de commerce le dispositif rappelle que les dispositions « ne font pas obstacle à la faculté de résiliation sans préavis, en cas d'inexécution par l'autre partie de ses obligations ou en cas de force majeure ».

${ }^{42}$ CA Paris, Pôle 5, ch. 4, 15 avr. 2015, n 13/02675.
} 
Dualité de flux. Deux arrêts ont examiné la relation entre le caviste et les courtiers ${ }^{43}$. L'activité de courtier, qui consiste à rapprocher les deux parties en vue de la conclusion de la vente, constitue une activité de prestation de services qui entre dans le champ d'application du texte ${ }^{44}$. Dans une première affaire $^{45}$, la société Bordeaux Magnum entretenait une relation commerciale avec un courtier selon deux flux bien distincts. Selon un premier flux, la société Bordeaux Magnum achetait des vins aux producteurs, en primeur, par l'intermédiaire du courtier. Selon le second flux, la société Bordeaux Magnum vendait des vins par l'intermédiaire du courtier. L'arrêt nous apprend que ces deux relations ont connu un développement notable entre 2004 et 2007, puis qu'elles ont, depuis le mois de juillet de l'année 2007, à l'exception d'achats en primeur du fait de la société Bordeaux Magnum ; qu'elles ont repris faiblement sur l'année 2011. La société Bordeaux Magnum soutenait ainsi qu'elle avait été victime d'une rupture brutale des relations commerciales de la part du courtier, et ce sans le moindre préavis. Examinant l'affaire au regard de la double qualité d'acheteur et de vendeur du requérant, la Cour appréciait le sort de ces deux courants d'affaires. Elle soulignait d'une part l'absence de commandes du caviste par l'intermédiaire du courtier, d'autre part son inertie face à l'absence de demandes d'achats présentées par le courtier pour ses clients, le caviste ne s'étant manifesté que cinq ans après la baisse des relations, si bien que pour les deux relations commerciales la preuve d'une rupture brutale n'était pas rapportée. Dans une seconde affaire ${ }^{46}$ le caviste avait assigné un autre courtier avec lequel il entretenait des relations similaires de vente consistant en l'achat par le courtier de vins pour le compte de ses clients et d'achats de vin par le caviste à des propriétés du bordelais par l'intermédiaire du courtier. Après un développement soutenu pendant trois années, ces deux relations avaient brutalement cessé en 2008. S'agissant des achats en primeur du caviste par l'intermédiaire du courtier, la demande était rejetée pour plusieurs raisons. Le caviste avait lui-même laissé sans réponse des propositions de vins, le courtier insistant par ailleurs sur le fait que certains châteaux avaient eux-mêmes décidé de ne pas allotir le caviste. La Cour rappelait « que l'allocation de primeurs reste à la discrétion du viticulteur et que le courtier comme le négociant ne peut être responsable de la décision de celui-ci. S'agissant en revanche des achats au caviste par le courtier, la Cour constate que passée une certaine date aucune commande n'avait été passée par le courtier, ce qui était bien caractéristique d'une rupture brutale illustrée par le volume d'affaires conséquent de la dernière année de collaboration commerciale (plus de 500000 euros), pour un préjudice de presque 180000 euros.

\section{L'action contre le négociant}

Imputation de la rupture. Le caviste avait enfin mené une action contre un négociant primoacquéreur ${ }^{47}$ en qualité de second acheteur, rejetée faute d'avoir tissé une relation commerciale suffisamment intense. La Cour avait jugé que nonobstant les commandes ponctuelles de vins primeurs à raison de 6 et 9 caisses passées en 2007 et 2008, il n'était pas démontré que la société Bordeaux Magnum ait tissé une relation continue d'une certaine intensité avec la société qui s'inscrive dans la durée et avait ainsi adopté les motifs des premiers juges qui avaient estimé que l'existence de relations commerciales établies entre les deux sociétés n'était pas démontrée. La Cour avait complété sa motivation jugeant en outre qu'en l'absence de toute convention entre les parties et compte tenu des pratiques habituelles et connues des professionnels dans le domaine de la vente des vins en primeur, il appartenait à la société Magnum de se manifester auprès de la société pour passer commande en 2010 des primeurs millésimés 2009 et « qu'aucune obligation de proposer ses vins à la vente en " primeur » n'est établie à l'encontre du négociant, si bien qu'en n'effectuant

\footnotetext{
${ }^{43}$ CA Paris, Pôle 5, ch. 5, 17 déc. 2015, n 14/11798 ; CA Bordeaux, 2ème ch. civ., 9 févr. 2011, nº 09/04160.

${ }^{44}$ C.-A. Maetz, fasc. préc., $\mathrm{n}^{\circ} 16$.

${ }^{45}$ CA Paris, Pôle 5, ch. 5, 17 déc. 2015, nº 14/11798.

${ }^{46}$ CA Bordeaux, $2^{\text {ème }}$ ch. civ., 9 févr. 2011, nº 09/04160.

47 CA Paris, 23 juin 2016, n 14/21935.
} 
aucune démarche "proactive auprès de la société Bordeaux Magnum » la société négociante n'avait commis aucune faute ${ }^{48}$.

\section{Les enseignements}

Apports en droit de la vente de vin. Ces arrêts confirment un certain nombre d'acquis jurisprudentiels du régime de responsabilité pour rupture brutale des relations commerciales établies, qu'il s'agisse du défendeur (professionnel civil ou commerçant) comme du caractère de la relation (on entend par là commerciale ou mixte). La difficulté propre aux cas d'espèce résidait dans la caractérisation de l'existence de la relation et de l'examen de la rupture, ce qui supposait un examen analytique de la vente en primeur, élément par élément (A), ainsi qu'une analyse contextuelle de cette vente en la situant notamment dans son environnement normatif local $(\mathbf{B})$. Ce sont ces deux aspects de droit du vin que nous allons développer ${ }^{49}$.

\section{A. La compréhension analytique de la vente en primeur}

Approche objective et subjective. La réparation de la rupture brutale de la relation commerciale établie suppose, entre autres conditions, la preuve d'une part de l'existence d'une relation commerciale établie et, d'autre part, la preuve d'une rupture caractérisée par sa brutalité. Dans les arrêts la distinction de ces deux conditions n'est pas toujours franche. En reprenant chronologiquement l'affaire Bordeaux Magnum, on peut d'ailleurs voir évoluer la défense des demandeurs. Si dans un premier temps l'accent a été mis sur l'analyse de la rupture au regard de la spécificité de la prestation contractuelle, c'est ensuite l'existence d'une relation commerciale établie qui a été directement remise en cause. Dans ces discussions, deux éléments ont occupé un rôle central : l'allocation (1) et le statut professionnel des intervenants à l'opération (2).

\section{L'allocation}

L'aléa climatique, fondement du système de l'allocation. Si le climat fournit au terroir viticole l'un de ses principaux facteurs naturels, l'aléa climatique demeure une donnée essentielle d'une grande partie de l'agriculture et de son traitement juridique. Cette contrainte naturelle de la production et ses conséquences sur un contrat de vente de vin avaient déjà été relevées dans un arrêt de la Cour d'Aix-en-Provence dans une affaire de refus de vente ${ }^{50}$ entre professionnels, pour caractériser l'impossibilité matérielle de fournir, "du vin provenant d'un château », s'il est «manifeste que la production ne permettrait pas de livrer l'ensemble des détaillants qui en feraient la demande ». Dans le contentieux étudié, cet aléa constitue le fondement économique de l'allocation, les arrêts soulignant que cet aléa climatique ne permet pas de garantir d'une année sur l'autre la même quantité de vin de sorte que les ventes sont réalisées selon un système d'allocations variables chaque année. L'aléa climatique se trouve donc identifié comme fondement de l'allocation au sens ou la quantité de vin disponible fluctuant chaque année au gré de différents facteurs, climatiques comme qualitatifs, la proposition faite aux acheteurs par l'intermédiaire des courtiers demeure nécessairement variable. L'aléa climatique a également été mobilisé de manière très circonstanciée, en complément d'autres facteurs ${ }^{51}$ pour établir la dégressivité des quantités allouées, et donc in fine pour caractériser la précarité de la relation contractuelle ${ }^{52}$. Il nous paraitrait plus exact

\footnotetext{
48 Rapp. E. Agostini, «Primeurs en Bordelais », art. préc. : « dès lors, si le négociant n’est pas avisé de son allocation avant le départ des primeurs, c'est à lui de s'émouvoir et de demander des explications directement ou par l'intermédiaire du courtier qui jouera les bons offices sans rien pouvoir contre la décision du propriétaire». ${ }^{49}$ C.-A. Maetz, fas. préc., $n^{\circ}$ 26. Comp. G. Chantepie, «La précarité des relations commerciales », Contrats, conc. consom., nov. 2012, étude 11, qui distingue nature de la relation et contexte de la relation.

${ }^{50}$ CA Aix en Provence, 24 avr. 1991, n 89-8853.

${ }^{51}$ Activité professionnelle de caviste du demandeur et, selon la Cour, « nature de la prestation en cause ».

52 V. infra
} 
de considérer que, envisagé de manière autonome, l'aléa climatique explique la variabilité de l'allocation, c'est-à-dire que cet aléa peut justifier une baisse des allocations et évincerait à ce titre toute responsabilité pour diminution des quantités allouées, ce qui correspond à une rupture partielle. En revanche, la précarité de la relation contractuelle doit être trouvée dans la nature intuitu personae du contrat.

L'allocation, offre de contrat intuitu personae. Il est remarquable que soit souligné dans deux arrêts que «que l'allocation de primeurs reste à la discrétion du viticulteur " $^{53}$, quoiqu'aucune conséquence juridique ne soit directement tirée de cette affirmation. Cette analyse est conforme au fait que le château emprunte au négociant ses structures de distribution. Ce faisant, le château inclut nécessairement des éléments tenant à la personne du négociant dans la relation contractuelle, ce qui établit la qualification intuitu personae de la vente. La vente en primeur constituant à la fois une opération de distribution sélective et un mode de financement ${ }^{54}$, il est incontestable que le château est attentif à la personne de son acheteur. Dans le cadre d'une revente ${ }^{55}$, le vendeur porte également une attention particulière à la personne du sous-acquéreur. L'on sait par exemple que la revente des crus à la grande distribution demeure un sujet sensible, comme l'illustre bien un arrêt rendu à propos d'un contrat-cadre entre une grande maison rhodanienne et un revendeur ${ }^{56}$. Sur le plan de la technique juridique, le château peut donc se prévaloir de la liberté exprimée par la notion d'allocation, qui n'est rien d'autre, au plan civil, qu'une offre de contrat intuitu personae ${ }^{57}$ : l'allocation constitue la proposition de se porter acquéreur, proposition qui contient les éléments essentiels du contrat intuitu personae proposé (chose et prix). Le caractère intuitu personae de la première vente résulte des obligations particulières de faire et de ne pas faire qui pèsent sur le négociant. A côté du paiement du prix pèse en effet sur l'acquéreur une obligation de faire, valoriser le cru, et une obligation de ne pas faire, consistant à ne pas porter atteinte au prestige du cru. On trouve ainsi dans les bordereaux de courtage des clauses selon lesquelles «la propriété se réserve le droit d'annuler la présente vente, en tout ou partie, si elle estime devoir prendre cette mesure dans l'intérêt de la réputation de son cru ». En pratique les négociants sont hiérarchisés par les châteaux, "évalués et catégorisés chaque année par les propriétés les plus prestigieuses, qui notent généralement la qualité du travail effectué selon cinq critères : l'implication du négociant pour la marque et la qualité de sa distribution (création d'évènements prestigieux, nouveaux marchés bien choisis...) sa solvabilité financière, la qualité du stockage des vins, le volume d'achat des vins livrables au cours de l'année passée, et la transparence relative à la distribution des crus ${ }^{58}$.

La fonction de l'allocation dans la rupture brutale des relations commerciales établies. Au cas précis ou interviendrait cette cessation de la relation contractuelle constituée par des ventes annuelles successives en primeur, quel pourrait être l'effet de l'intuitus personae? Envisageons successivement, en suivant la chronologie des arrêts, son effet possible sur la rupture puis sur la qualification de la relation commerciale établie.

Allocation et rupture de la relation commerciale. Le caractère intuitu personae n'a donc pas vocation à exclure la responsabilitée ${ }^{59}$, mais sert à asseoir la prévisibilité de la rupture, ce qui éloigne sa brutalité, par exemple dans l'hypothèse où le producteur aurait reproché au négociant sa

\footnotetext{
${ }^{53}$ CA Bordeaux, 2ème ch. civ., 2 juill. 2015, nº 10/07300.

${ }^{54}$ Comp. E. Agostini, « Primeurs en Bordelais », art. préc.

55 V. supra

56 Cass. com., 18 déc. 2012, n 11-27.342.

${ }^{57}$ V. E. Agostini, « Primeurs en Bordelais », art. préc., qui évoque un « acte de pure faculté ».

58 P.-M. Chauvin, Le marché des réputations, Une sociologie du monde des vins de Bordeaux, op. cit., p. 71.

${ }^{59}$ V. G. Chantepie, «La précarité des relations commerciales », art. préc., n 11 et les références citées.
} 
défaillance dans la valorisation du cru et un manquement à ses obligations de faire ${ }^{60}$. On remarquera que dans deux arrêts le producteur reprochait à son acheteur "de ne pas avoir valorisé sa production, de commercialiser les produits dans le but essentiel de se procurer de la trésorerie » si bien que «ces faits et le tour que semblent prendre [les] relations [étaient] susceptibles de compromettre définitivement pour l'avenir la poursuite [des] rapports commerciaux ${ }^{61}$. Rappelons que l'article L. $442-6$, I, $5^{\circ}$ prévoyait ${ }^{62}$ que "les dispositions qui précèdent ne font pas obstacle à la faculté de résiliation sans préavis, en cas d'inexécution par l'autre partie de ses obligations ou en cas de force majeure ».

Allocation et existence de la relation commerciale. L'allocation pourrait ensuite miner tout espoir de reconduction automatique, fragilisant par-là la relation commerciale en l'affectant d'une cause intrinsèque de précarité. Précisément, c'est un fait plutôt admis dans la filière que l'allocation de vin en primeur est à la discrétion du producteur, si bien que l'allocataire ne bénéficierait d'aucun droit acquis à la reconduction, ce qui est une manière d'exprimer que l'allocation constitue bien une offre de contrat intuitu personae, et procède à ce titre d'une manifestation unilatérale de volonté. Selon Éric Agostini, en effet : « dans la pratique bordelaise, à part la commande et le règlement de l'acquéreur, le seul écrit qui matérialise l'achat de l'allocation par le négociant est le bordereau de courtage. Or, il n'y est jamais question d'une quelconque préférence pour l'année suivante. Le viticulteur est seul maitre du jeu. L'octroi de l'allocation est à sa discrétion et elle ne lie pas le négociant. Autrement dit, le négociant peut acheter, ne pas acheter ou moins acheter. Le producteur peut allouer, moins allouer ou ne rien allouer du tout ». Il ressort effectivement des arrêts étudiés que l'intuitus personae va contribuer, avec d'autres éléments (aléa climatique et profession de caviste) à la preuve de la précarité de la relation contractuelle en rejoignant l'hypothèse déjà admise en jurisprudence selon laquelle lorsque les usages de la profession excluent toute pérennité, avec clause de non-reconduction tacite, le cocontractant ne peut légitimement s'attendre à la stabilité de la relation ${ }^{63}$.

\section{L'incidence des statuts professionnels sur l'existence de la relation commerciale}

Prise en compte des acteurs professionnels. Le schéma classique et tripartite de la vente en primeur associe producteurs, courtiers et négociants. Ce contentieux a bien mis en lumière la stricte répartition des rôles.

La situation du courtier. Rappelons au sujet des courtiers que la naissance d'un commerce de place $^{64}$ a structuré le commerce du vin en amont, donnant naissance à une association puissante entre viticulteurs, courtiers ${ }^{65}$ et négociants. Economiquement, les courtiers sont ainsi chargés de rapprocher les maillons extrêmes de la chaîne. Cela consiste «à faire connaître à chaque partie les conditions de l'autre, les renseigner sur la situation du marché et les conseiller, tenter de concilier leurs intérêts éventuellement divergents ${ }^{66}$. Juridiquement, le rôle du courtier en vin consiste donc à rapprocher viticulteurs et négociants en vue de conclure un contrat de vente de vins, le cas échéant

${ }^{60}$ CA Paris, Pôle 5, ch. 4, 15 avr. 2015, préc. ; CA, Paris, Pôle 5, ch. 4, 15 avr. 2015, n 13/02675 : la Cour rejette la brutalité de la rupture au seul constat d'une lettre du château reprochant au caviste « de ne pas avoir valorisé sa production ».

61 Ibid.

62 V. aujourd'hui C. com., art. L. 442-1, II.

${ }^{63}$ Cass. com., 18 mai 2010, $\mathrm{n}^{\circ}$ 08-21.681 : selon la chambre commerciale, il appartient aux juges du fond de « rechercher si, eu égard à la nature de leurs prestations [...], les sociétés plaignantes pouvaient légitimement s'attendre à la stabilité de leur relation...».

${ }^{64}$ V. supra.

65 T. Favario, «Courtage et négoce de vin », RD rur. 2017, n 11, p. 28 et s. ; J.-M. Bahans et M. Menjucq, Droit de la vigne et du vin, aspects juridiques du marché viticole, op. cit., préc., $\mathrm{n}^{\mathrm{os}} 127$ et s. et $\mathrm{n}^{\text {os }} 827$ et s.

${ }^{66}$ O. Antoine-Geny et M. Roudil, Manuel pratique du commerce des vins, op. cit., p. 35. 
en primeurs. Chargé en toute hypothèse d'opérer une intermédiation, le courtier n'occupe cependant pas la même situation selon que le demandeur à l'action en réparation pour rupture brutale de la relation commerciale agit comme acheteur allocataire ou comme vendeur de grands crus. Lorsque le caviste agissait comme premier acheteur, auprès des châteaux, il a été retenu que le courtier demeurait dépendant du bon vouloir du vendeur, attentif aux réseaux de distribution que son cru emprunte, ce qui protégeait le courtier qui pouvait se retrancher derrière la volonté du château ${ }^{67}$. Quand bien même, en pratique, les quantités allouées à chaque négociant seraient conjointement définies par les courtiers et les producteurs, la décision finale appartient au château, si bien que le courtier bénéficie de la même protection qu'un professionnel dépendant des possibilités de son fournisseur ${ }^{68}$. En revanche, si le demandeur à l'action est vendeur, le courtier retrouve une certaine liberté et s'expose à une condamnation en cas de rupture brutale de la relation commerciale établie s'il ne sollicite plus le caviste pour le compte de ses clients ${ }^{69}$.

La situation du caviste. En matière de rupture brutale des relations contractuelles d'affaires, la précarité subjective est généralement liée à une procédure d'appel d'offres ${ }^{70}$. Ici elle tient à la profession de l'acheteur. Lorsque ces litiges sont nés, la jurisprudence était déjà bien fixée quant à la caractérisation de la relation commerciale établie ${ }^{71}$, laquelle exige un caractère suivi, stable et habituel. La partie victime de l'interruption doit pouvoir raisonnablement anticiper pour l'avenir une certaine continuité du flux d'affaires avec son partenaire commercial ${ }^{72}$. Cela confère un rôle central à la croyance légitime de la victime de la rupture dans la pérennité de la relation, « elle-même analysée à l'aune d'un faisceau d'indices appréciés in concreto parmi lesquels, essentiellement, la durée, la continuité et surtout la stabilité de la relation commerciale $»^{73}$. Le défendeur à l'action devait donc établir tout élément de précarité ${ }^{74}$ de la relation, susceptible d'écarter la qualification de relation commerciale établie. Malgré un statut commun de professionnel commerçant, négociants et cavistes ne peuvent effectivement être assimilés sur le plan économique. Comme les cavistes le relèvent eux-mêmes sur le site de la profession, la tradition institutionnelle viticole française a historiquement structuré les activités de production en amont, côté viticulture, en intégrant deux professions, les négociants et les courtiers ${ }^{75}$, mais elle n'a pas intégré les métiers de l'aval, ceux de la commercialisation aux clientèles finales. Ces commerçants, boutiquiers ordinaires au contact des consommateurs, ne justifiaient pas l'existence de règles spécifiques. Il en résulte que le caviste n'est pas un vrai professionnel de la place comme le sont les négociants et les courtiers. L'achat direct par le caviste s'apparente donc à un mode inhabituel de distribution qui peut rendre plus difficile la qualification de relation commerciale, relation revêtue d'un caractère exceptionnel et par là précaire $^{76}$. Il s'agit ici d'une précarité liée au contexte - le commerce de place - de la relation contractuelle $^{77}$. A ce sujet, il nous semble que le raisonnement aurait pu être mené à l'avantage de l'acheteur, car une fois entré dans le cercle des allocataires il n'y a pas de raison particulière d'apprécier singulièrement l'attente légitime du caviste. Si l'on devait conférer à cette règle la force

\footnotetext{
${ }^{67}$ P.-M. Chauvin, Le marché des réputations, Une sociologie du monde des vins de Bordeaux, op. cit., p. 78.

68 Rapp. Cass. com., 13 mars 2013, n 12-25.361 : JurisData $\mathrm{n}^{\circ}$ 2013-025587 : Contrats, conc. consom. 2014, comm. 40, note N. Mathey, reprochant aux juges du fond de ne pas avoir recherché si «eu égard à la nature de la prestation, qui dépendait des commandes obtenues par la société LJF auprès de différents industriels de l'automobile, la société MTLS pouvait légitimement s'attendre à a stabilité de sa relation avec la société LJF ».

${ }^{69}$ CA Bordeaux, 2ème ch., 9 févr. 2011, nº 09/04160.

${ }^{70}$ C.-A. Maetz, fasc. préc., $\mathrm{n}^{\circ} 27$.

${ }^{71}$ Sur lesquels v. C.-A. Maetz, fasc. préc., $\mathrm{n}^{\circ} 22$.

${ }^{72}$ Cour de cassation, Rapport annuel 2008, p. 307, à propos de Cass. com., 16 déc. 2008, n 07-15.589, Bull. civ. IV, n 207 ; Contrats, conc. consom. 2009, comm. 73, note N. Mathey.

73 C.-A. Maetz, fasc. préc., $\mathrm{n}^{\circ} 22$.

${ }^{74}$ G. Chantepie, «La précarité des relations commerciales », art. préc.

75 T. Favario, «Courtage et négoce de vin », art. préc.

${ }^{76}$ N. Mathey, «La rupture n’était pas brutale », Contrats, conc. consom. 2015, n 7, comm. 174.

77 G. Chantepie, «La précarité des relations commerciales », art. préc.
} 
d'un usage, ce qui pourra être vérifié ${ }^{78}$, cette allocation traduit une volonté des producteurs de déroger à la règle, caractérisant une renonciation tacite à s'en prévaloir. La situation est en effet paradoxale : peut-on à la fois opposer au caviste un usage de place et lui expliquer qu'il n'intègre pas la place ? En outre, si la distinction entre caviste et négociant existe économiquement, elle est beaucoup moins nette en droit. Il n'existe pas en effet de statut spécifique du négociant, mais un regroupement par le truchement d'un syndicat professionnel via un système de cooptation. Pour bénéficier de la vente en primeur, faut-il être négociant ou faut-il, comme en l'espèce, exercer une activité de négoce?

\section{B. La compréhension structurelle de la vente en primeur}

La réception juridictionnelle des usages et pratiques de place. La figure contractuelle de la vente en primeur résulte avant tout d'une pratique de place, déterminée par des considérations économiques et par l'histoire. Il est à ce titre particulièrement intéressant d'apprécier comment la technique juridique reçoit les habitudes de place pour les traduire, sur le plan contractuel, ce à quoi correspond l'usage défini comme des «comportements dotés d'une force juridique en raison de leur répétition et leur généralité» ${ }^{79}$ les usages s'épanouissent de manière polymorphe en matière viticole $^{80}$. En amont, côté production, les usages ont fourni un support à la détermination des aires et des méthodes de production. En aval, on les retrouve principalement en droit de la vente, en matière d'agréage et d'intermédiation du courtier. Ils sont parfois repris par les règlementations professionnelles locales ${ }^{81}$. L'on assiste dans le contentieux ici étudié à la consécration d'un usage de place, au même titre que ceux gouvernant l'intervention du courtier, à ceci près qu'ils ne viennent pas directement encadrer la formation du contrat, mais plutôt aider à la mise en œuvre de dispositions légales gouvernant la fin des relations commerciales. Dans ce contentieux, l'usage apparaissait en effet à plusieurs reprises. Côté demandeur, il était allégué l'existence d'un usage relatif à la reconduction de l'allocation d'une année sur l'autre et la nécessité de respecter un préavis d'un an. Côté défendeur, c'est l'usage réservant aux négociants les achats en primeur qui a été plaidé. Côté magistrats, l'usage a été expressément mobilisé pour apprécier l'existence de la relation commerciale, celle-ci « devant être examinée au regard des usages en matière de vente de vins en primeur, ainsi qu'au regard du produit vendu ». Le recours à l'usage pour établir la précarité de la relation commerciale établie avec le caviste non négociant ne repose pas à proprement parler sur usage conventionnel qui gouvernerait les relations contractuelles, mais sur un usage de marché ${ }^{82}$, utilisé ici pour établir le caractère inhabituel et par là précaire d'une relation établie sur une place considérée. Ici, l'usage de marché concerne l'incidence d'un contexte sur une relation contractuelle. Dans le même registre, mais cette fois-ci pour imputer la rupture, dans l'arrêt ayant rejeté la demande du caviste contre un négociant, le juge s'est aussi inspiré des "pratiques» locales, qui commanderaient que le négociant se rapproche du producteur lorsqu'il constate qu'il est n'est plus alloti $^{83}$. L'on soulignera d'ailleurs que seule la première vente est ainsi marquée par l'importance de

\footnotetext{
78 V. infra.

${ }^{79}$ P. Mousseron, «Introduction terminologique : des comportements aux "bonnes pratiques", Journal des sociétés, novembre 2011, p. 12 et s.

80 Sur les usages en matière viticole, v. notamment P. Mousseron, «Les usages relatifs au vin: Nunc est bibendum », DESC (Direito, Economica e Socidedade Contemporanea), vol. 1, n 1, p. 176 et s.

81 V. J.-M. Bahans et M. Menjucq, Droit de la vigne et du vin, aspects juridiques du marché viticole, op. cit., n $\mathrm{n}^{\mathrm{os}} 787 \mathrm{et} \mathrm{s}$. et nos 827 et $\mathrm{s}$.

82 P. Mousseron, «Introduction terminologique : des comportements aux "bonnes pratiques”, art. préc., p. 12.

${ }^{83}$ CA Paris, 23 juin 2016, n 14/21935 : « c'est à juste titre, par des motifs que la cour adopte, que les premiers juges ont estimé que l'existence de relations commerciales établies entre les deux sociétés n'était pas démontrée ; Considérant en outre qu'en l'absence de toute convention entre les parties et compte tenu des pratiques habituelles et connues des professionnels dans le domaine de la vente des vins en primeur, il appartenait à la société Magnum de se manifester auprès de la société Duclot pour passer commande en 2010 des primeurs millésimés 2009 ; Qu'aucune obligation de proposer ses vins à la vente en "primeur" n'est établie à l'encontre de la société Duclot; Qu'en ne faisait pas de démarche proactive auprès de la société Bordeaux Magnum, la société
} 
la « place » et l'intégration de la tradition économique et aux usages juridiques. Ce n'est pas le cas de la seconde, qu'il s'agisse de l'intermédiation du courtier, du formalisme contractuel qui préside à l'élaboration de la transaction ou de l'allocation. C'est cette indépendance qui a permis au caviste d'engranger quelques succès dans les contentieux portant sur des relations commerciales dans lesquelles il intervenait comme vendeur ou comme second acquéreur.

Les relations de place. Pour illustrer la force des relations de place, l'on portera à la connaissance du lecteur un fait important. Le caviste avait signé le 25 mai 2007 une promesse de bail commercial avec le Grand Hôtel de Bordeaux. La promesse portait sur l'occupation de locaux dépendant de la galerie du Grand Hôtel en vue de leur exploitation comme cave à vins. Le bail a été signé le 24 septembre 2007. Le 11 septembre 2014, la Cour d'appel de Bordeaux a annulé ce bail sur le fondement d'une réticence dolosive de Bordeaux Magnum. L'arrêt de la Cour de Bordeaux retient que « la société Bordeaux Magnum a dissimulé volontairement au bailleur qu'elle était engagée dans un litige portant sur une pratique locale », le contentieux initié contre le Château Latour (qui a depuis quitté les primeurs) «que cela excluait tout partenariat entre elle et la société Château Latour dont les vins sont parmi les plus réputés du bordelais, et, partant avec le bailleur, voire d'autres châteaux compte tenu de la nature du litige » et que dès lors « ce litige était en totale contradiction avec le projet de partenariat conçu par la société bailleresse avec les viticulteurs bordelais de renom ». L'arrêt en déduit que, par ce silence, la société Bordeaux Magnum a dissimulé à son cocontractant un fait qui, s'il avait été connu de lui, l'aurait empêché de contracter. Le pourvoi contre cet arrêt a été rejetét ${ }^{84}$.

Duclot n'a commis aucune faute ». Rapp. E. Agostini, «Primeurs en Bordelais », art. préc. : " dès lors, si le négociant n'est pas avisé de son allocation avant le départ des primeurs, c'est à lui de s'émouvoir et de demander des explications directement ou par l'intermédiaire du courtier qui jouera les bons offices sans rien pouvoir contre la décision du propriétaire.

${ }^{84}$ Cass. com., 7 juin 2016, $\mathrm{n}^{\circ} 14-27.264$. 